Methods Transcriptomic analysis using bulk RNA sequencing was performed on inflamed $\mathrm{CD}\left(\mathrm{n}_{\mathrm{CD}}=4\right)$ and non-IBD control $\left(\mathrm{n}_{\mathrm{NC}}=6\right)$ colonic biopsies taken during colonscopy. Raw gene expression data were processed using an RNA sequencing exploration pipeline, and immune system-associated genes and pathways were selected to assess immunopathotypes. To validate findings, publicly deposited gene expression datasets were used and targets of interest quantified using immunohistochemistry (IHC) $\left(\mathrm{n}_{\mathrm{CD}}=23\right.$ and $\left.\mathrm{n}_{\mathrm{NC}}=22\right)$.

Results Our transcriptomic analyses identified four enriched clusters linked to different immune pathways and cell types in distinct subsets of CD patients with ongoing inflammation. Validation in a larger gene expression dataset highlighted an immune cluster characterised by macrophage and neutrophil activation and infiltration. IHC analysis demonstrated the colocalisation of proteins encoded by signature genes with the macrophage marker CD68, suggesting that macrophages are the key cell type. Our findings indicated the presence of two macrophage subsets that are potentially interacting to perpetuate inflammation in a subgroup of $\mathrm{CD}$ patients. The clinical relevance of this signature is currently still being evaluated to explore if it is a suitable biomarker of a distinct CD immunopathotype.

Conclusions Overall our data shows a unique macrophageassociated signature to be enriched in a subset of CD patients, the potential clinical utility of which is currently under investigation.

\section{PMO-2 CAN WE PREDICT CMV COLITIS WITH HAEMOGRAM- BASED INFLAMMATORY INDICES?}

${ }^{1}$ Arda Yavuz*, ${ }^{1}$ Kubra Akan, ${ }^{2}$ Muhammet Mikdat Akbas, ${ }^{2}$ Busra Gulec, ${ }^{1}$ Celal Ulasoglu, ${ }^{1}$ llyas Tuncer. 'Istanbul Medeniyet University, Department of Gastroenterology, Istanbul, Türkiye; ${ }^{2}$ stanbul Medeniyet University, Department of Internal Medicine, Istanbul, Türkiye

\subsection{6/gutjnl-2021-BSG.141}

Introduction This study aims to assess the predictive value of indices as systemic immune-inflammation index (SII), neutrophil-lymphocyte ratio (NLR) and monocyte/lymphocyte ratio (MLR) on presence of active CMV infection in UC patient.

Methods We retrospectively reviewed colonoscopy reports of UC patients between January 2011 and January 2021. These indices are derived from the $\mathrm{CBC}$ parameters. CMV colitis was diagnosed from PCR of colonic tissue. The patients with CMV colitis were treated with gancyclovir for 21 days. Statistical analyses were performed using SPSS. Receiver operating characteristic (ROC) curve analysis was used to determine the cut-off values for predicting CMV colitis.

Results A total of 269 UC patients were involved. 19 (7.1\%) patients had documented CMV colitis. CMV patients had pancolitis $(n=11,57.9 \%)$ and left-sided colitis in $8(42.1 \%)$. The drugs used were mesalazine monotherapy $(n=9)$, azathioprine $(n=6)$ and anti-TNF therapy $(n=4)$. The control group consisted of 250 UC patients without active CMV. This group mostly had left-sided colitis $(n=128)$, while the number of proctitis was 40 and pancolitis in 82 .

There were significant differences between CMV PCR $(+)$ and CMV PCR (-) cases by means of SII $(p=0,037)$, NLR $(p=0,040)$ and MLR $(p=0,016)$ indices. Moreover, there was no significant superiority between SII, NLR, and MLR. According to the ROC curve analysis, the best cut-off MLR value to differentiate between patients with CMV colitis from UC was >0.43 (Sens:36.8 ;Spec:89.1 ; PPV:21.2 ; NPV:94.7),
Abstract PM0-2 Table 1 Comparison of indices and cut off values according to CMV PCR

\begin{tabular}{|c|c|c|c|}
\hline & $\begin{array}{l}\text { CMV PCR } \\
(+)\end{array}$ & $\begin{array}{l}\text { CMV PCR(- } \\
\text { ) }\end{array}$ & $P$ value \\
\hline $\begin{array}{l}\text { Total } \\
(\mathrm{n}=269)\end{array}$ & 19 & 250 & NA \\
\hline Gender F/M & $5 / 14$ & $105 / 145$ & 0.169 \\
\hline SII $<900$ & 6 & 135 & $0.036^{*}$ \\
\hline SII >900 & 13 & 104 & \\
\hline NLR<3.1 & 6 & 138 & $0.027^{*}$ \\
\hline NLR $>3.1$ & 13 & 101 & \\
\hline$M L R<0.43$ & 12 & 213 & $0.005^{*}$ \\
\hline MLR $>0.43$ & 7 & 26 & \\
\hline
\end{tabular}

best cut-off NLR value was >3.1(Sens:68.4 ;Spec:57.7 ; PPV:11.4 ; NPV:95.8), and best cut-off SII value was $>900$ (Sens:68.4 ;Spec:52.3 ; PPV:11.1 ; NPV:95.7) (Table 1).

Conclusions This is the first report to demonstrate that SII, NLR, MLR may predict superinfection of CMV colitis in UC patients. The cut-off values were 900, 3.1 and 0.43 for systemic inflammation index, NLR and MLR, respectively. Thus, CMV complicating UC seems to result with higher inflammatory indices. Large scale, prospective studies are needed for further conclusion.

\section{PMO-3 THE SPIT STUDY: CREATING A SALIVA BASED EPIGENETIC BIOMARKER PANEL TO DIAGNOSE CROHN'S DISEASE}

${ }^{1}$ Timothy Stone*, ${ }^{1}$ Vanessa Ward, ${ }^{2}$ Avi Rosenfeld, ${ }^{1}$ Aine Hogan, ${ }^{3}$ Ami Ya'acov, ${ }^{3}$ Aviya Hoyda, ${ }^{3}$ Sapir Liansky, ${ }^{1}$ Ho K M A, ${ }^{1}$ Paul Wolfson, ${ }^{1}$ Ash Wilson, ${ }^{3}$ Ariella BarGal Shitrit, ${ }^{1}$ Elena Schiff, ${ }^{1}$ Adam Levine, ${ }^{4}$ The SPIT Study Group, ${ }^{1,5}$ Laurence Lovat. ${ }^{1}$ Division of Surgery and Interventional Science, University College London, London, UK; ${ }^{2}$ Department of Computer Science, Jerusalem College of Technology, Jerusalem, Israel; ${ }^{3}$ Digestive Diseases institute, Shaare Zedek Medical Center and Faculty of Medicine, Hebrew University of Jerusalem, Jerusalem, Israel; ${ }^{4}$ The SPIT Study Group; ${ }^{5}$ Department of Gastrointestinal Services, University College London Hospitals NHS Foundation Trust, London, UK

\subsection{6/gutjnl-2021-BSG.142}

Introduction The SPIT study (ISRCTN11921553) aims to investigate the methylation changes in saliva that result from Crohn's disease with the ultimate aim of developing a diagnostic biomarker.

Aims and Methods We selected 192 volunteers, 96 with Crohn's Disease and 96 controls. These were drawn from participants in the SPIT study and were carefully chosen so that differences in sex ratio and average age were as small as possible between the two groups. All participants completed a questionnaire on relevant lifestyle factors and provided a fasting saliva sample. Stringent DNA quality-control was performed. Following DNA extraction and bisulfite conversion, samples were analysed using the $850 \mathrm{k}$ Infinium methylation EPIC array.

Multiple quality-control metrics were analysed. This led to removal of 38 samples, leaving 154 samples for differential methylation analysis. Stringent batch-effect removal was applied by calculating the residuals of a batch-fitted linear model on each locus. Differential methylation analysis was 